\title{
SAND CASTING: CONVENTIONAL AND RAPID PROTOTYPING MANUFACTURING APPROACHES
}

\author{
Sagar M Baligidad ${ }^{1}$, N Krishnamurthy ${ }^{2}$, N Narendra ${ }^{3}$, Ajay Srinivasan $\mathbf{N}^{4}$ \\ ${ }^{1}$ Assistant Professor, Mechanical Engineering Department, CMR Institute of Technology, Bangalore, India \\ ${ }^{2}$ Professor, Mechanical Engineering Department, CMR Institute of Technology, Bangalore, India \\ ${ }^{3}$ Assistant Professor, Mechanical Engineering Department, CMR Institute of Technology, Bangalore, India \\ ${ }^{4}$ Research Scholar, Mechanical Engineering Department, CMR Institute of Technology, Bangalore, India
}

\begin{abstract}
Sand casting provides an economic method for mass production of complex and intricate features of metal parts. Manufacturing of moulds to cast small and medium type products involve relatively long lead time and high tooling costs. The application of Rapid Prototyping (RP) technologies to fabricate complex sacrificial patterns can result significant reduction in the cost and lead time associated with the manufacture of single, small or medium quantity productions. Previously this technology was used to develop a model of products. The current research work involves fabrication of pattern using Rapid Prototyping technique and same is used to produce metal castings. A comparison study has been done in the sense of lead time, dimensional accuracy, surface quality and cost investment of patterns and castings produced using RP technique and conventional method (using wooden pattern). It was observed that the patterns produced by the RP Technique have higher dimensional accuracy, good surface finish and nominal cost investment. Also the castings produced by this technique have dimensions, surface quality similar to that of pattern produced by this technique. It also reduces the manufacturing lead time.
\end{abstract}

Keywords: Sand casting, Rapid prototyping, Rapid tooling, Dimensional accuracy and Surface quality etc....

\section{INTRODUCTION}

Sand casting is one of the oldest metal manufacturing techniques, it employs wooden pattern to develop mould cavity by ramming the moulding sand around the pattern. The molten metal is poured in to the mould cavity and allowed for solidification. After the molten metal gets solidified, the mould is broken and the solid casting is removed [1].

Conventional sand casting method requires machine tools for the manufacturing of wooden patterns. Tooling cost depends on the complexity of the pattern. It is less for simple design but for complex design the tooling cost as well as time required to develop the design will be high. This conventional method is not economical when number of castings required is small in quantity.

Now there is an industrial need for manufacture of intricate castings, for defense, vintage equipment and medical prosthetics. Rapid Prototyping (RP) technique is a combined systematic approach which utilizes rapid tooling and internet technologies in manufacturing [2]. It involves automated fabrication of intricate shapes from Computer Aided Drafting (CAD) data using layer-by-layer principle. It allows designer to quickly create tangible prototypes of their designs. Development of intricate castings involves challenges of economic visibility, quick tooling and production of defect free castings in the first attempt. These challenges are overcome by employing RP technology which develops patterns for sand casting process. RP techniques can be used to develop complex patterns; also, the tool costs associated with the design and production of complex pattern will be avoided [3]. In conventional casting process, each iterative step requires substantial time and cost investments to modify the metal tooling. By employing RP fabricated pattern to one can reduce the iteration time and cost investment in tooling for single and small quantity productions.

The first use of RP models as a pattern started in the year 1989 [4, 5]. There are various types of commercially available RP techniques used to fabricate patterns, each with unique capabilities. These are Stereo lithography (SLA), Fused Deposition Modeling (FDM), Laminated Object Manufacturing (LOM), Selective Laser Sintering (SLS), Solid Ground Curing (SGC), Ink-Jet Printing (IJP) [6].

The main objective of this work is to produce sand castings of impeller of a centrifugal compressor using wooden and RP pattern and compare the dimensional accuracy, surface quality and cost investment of these castings.

\section{METHODOLOGY}

The procedure adopted to build the model in FDM rapid prototyping processes is termed as methodology. Methodology involves various steps to obtain the required outcome. Procedure has a logical sequence between the each activity, where the sequence defines the step-by-step approach towards the desired goal. Exact sequence of the task is essential failing which the required goal may not be achieved. The industries/foundries are therefore going towards the advanced techniques such as Computer Aided 
Design and Computer Aided Manufacturing (CAD/CAM). In this work, Computer Aided Design is used to generate the component part where as Rapid Prototyping is used for building pattern of impeller. The following procedure is applied in the production of RP pattern of impeller.

\subsection{CAD Model Creation}

First, the object to be built is modeled using a ComputerAided Design (CAD) software package. Solid modelers, such as Pro/ENGINEER, can develop 3-D objects more accurately than wire-frame modelers such as Auto CAD. The designer can use a pre-existing CAD file or may wish to create one separate file especially for prototyping purpose. This process is identical for all types of RP build techniques. The CAD and 3-D drawing of the impeller is shown in Fig. 2.1 and 2.2.

\subsection{Conversion to STL Format}

The various CAD packages use different algorithms to represent solid objects. To establish consistency, the STL (stereo lithography, the first RP technique) format has been adopted as the standard of the rapid prototyping industry. This format represents a three-dimensional surface as an assembly of planar triangles, "like the facets of a cut jewel." The file contains the coordinates of the vertices and the direction of the outward normal of each triangle. Because STL files use planar elements, they cannot represent curved surfaces exactly. Curvatures can be improved by increasing the number of triangles but it will increase the file size which requires more time to pre-process and build. Therefore the designer must balance accuracy with manageability to produce a useful STL file. Fig. 2.3 shows the conversion of CAD file into STL file.

\subsection{Slice the STL File}

In this step, a pre-processing program prepares the STL file to be built. Several programs are available, and most of them allow the user to adjust the size, location and orientation of the model. Built orientation is important for several reasons. Firstly, properties of rapid prototypes vary from one coordinate to another. For example, prototypes are usually weaker and less accurate in $\mathrm{z}$ (vertical) direction than in the $x-y$ plane. In addition, part orientation partially determines the amount of time required to build the model. Placing the shortest dimension in the $\mathrm{z}$ direction reduces the number of layers, thereby shortening build time. The preprocessing software slices the STL model into a number of layers from $0.01 \mathrm{~mm}$ to $0.7 \mathrm{~mm}$ thick, depending on the build technique (Fig.2.4). The program may also generate an auxiliary structure to support the model during the constuction (Fig. 2.5). Supports are useful for delicate features such as overhangs, internal cavities, and thin-walled sections.

\subsection{Layer by Layer Construction}

In this step, the actual impeller pattern is constructed using RP machine. Acrylonitrile Butadiene Styrene (ABS) polymer is used as a material for the constuction of
pattern.ABS material is a thermo setting plastic which gives a good surface finish to the pattern. Most of the RP machines are fairly autonomous, needing little human intervention.

\subsection{Cleaning and Finishing}

In this step, the pattern is removed from the machine and supports are detached. Patterns are subjected to surface treatment processes such as sanding, sealing, and/or painting to improve its appearance and durability. The actual pattern of the impeller is shown in Fig.2.6.

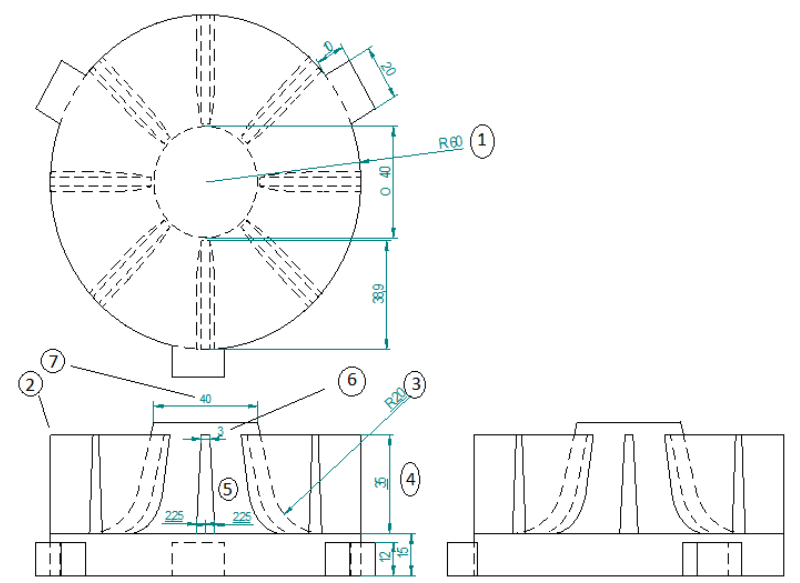

Fig -2.1: CAD drawing of benchmark design of impeller model.



Fig -2.2: 3-D model of the impeller



Fig -2.3: CAD file stored in .STL format 


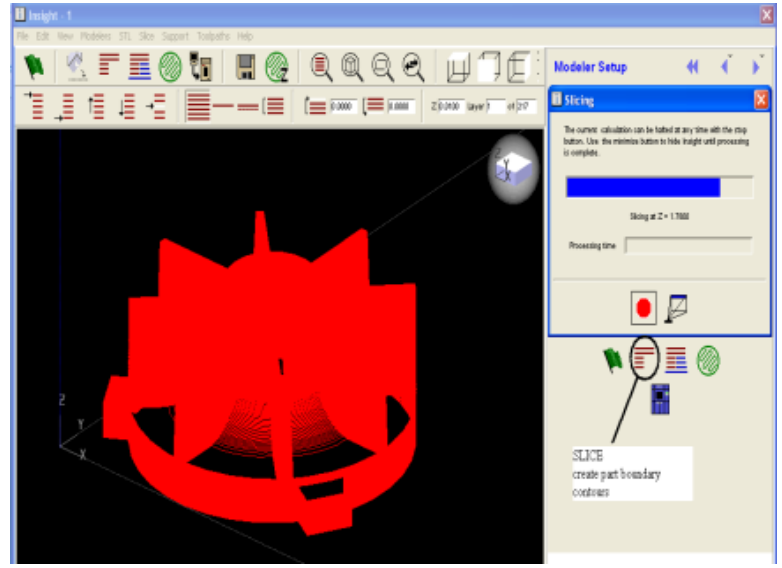

Fig -2.4: Slicing of the impeller model

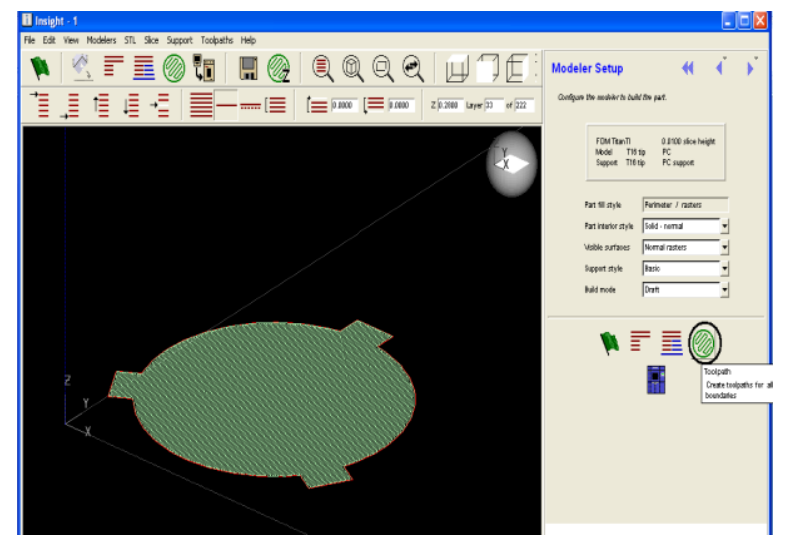

Fig -2.5: Generation of support material for impeller model using INSIGHT software

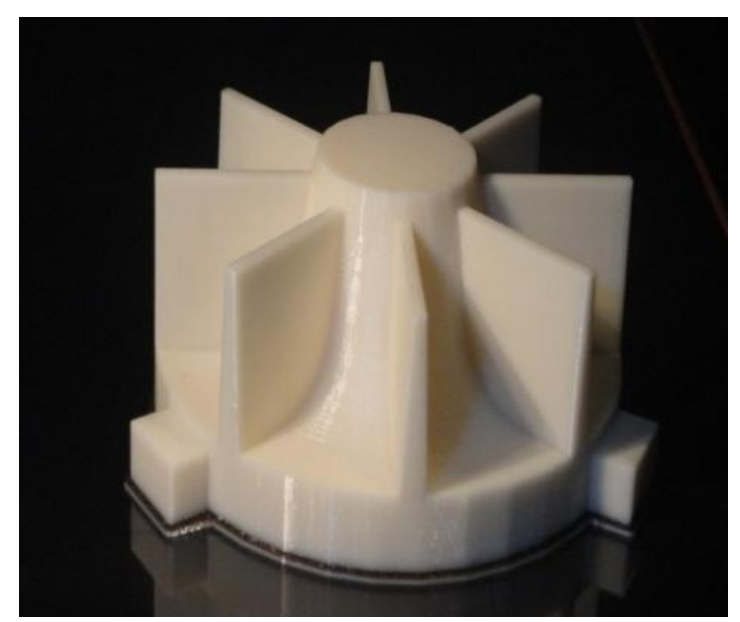

Fig -2.6 Actual ABS pattern of the impeller

\subsection{Casting of Impeller using Wooden and ABS}

\section{Patterns}

Castings of impeller is prepared using ABS pattern by applying actual foundry procedure. Aluminium is used as the material for cating. The casting of impeller using ABS pattern is shown in Fig. 2.7. Also aluminium impeller casting is produced using wooden pattern by applying actual foundry procedure. Fig. 2.8 and 2.9 shows the wooden pattern and casting of impeller.



Fig -2.7 Aluminium casting by ABS pattern

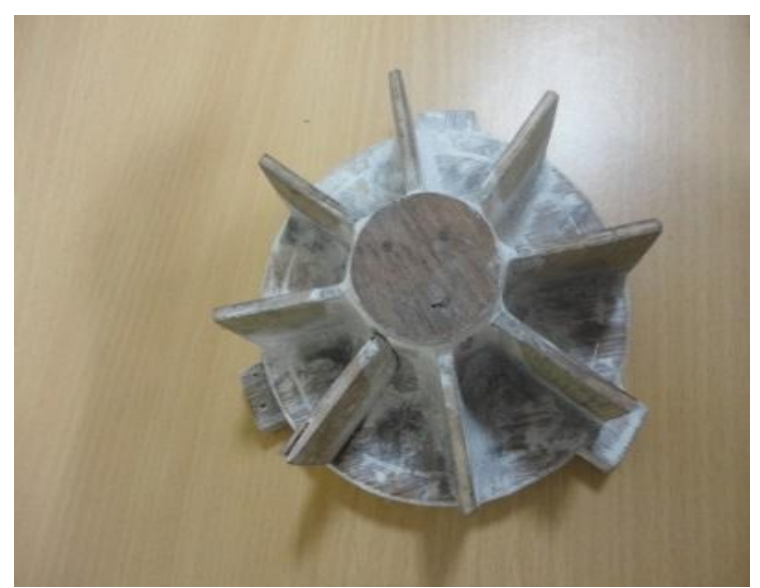

Fig -2.8 wooden pattern



Fig -2.9 Aluminium casting by wooden pattern

\section{COMPARISON OF CASTINGS PRODUCED} BY ABS AND WOODEN PATTERNS

\subsection{Dimensional Accuracy}

Some of the critical sections (Fig.2.1. indicated by numbers $1,2,3,4,5,6,7)$ are selected to examine the dimensional variations of ABS and wooden pattern castings. Dimensional distribution charts are plotted based on the measured values 
of wooden pattern and its castings as shown in Fig. 3.1 a and b. From the graphs, it is seen that $80 \%$ of dimensional deviations of the wooden pattern falls within $\pm 0.3 \mathrm{~mm}$ and the average dimensional deviation between ABS pattern and wooden pattern is approximately $0.025 \mathrm{~mm}$. For aluminum casting $80 \%$ of dimensional deviation falls within $\pm 0.3 \mathrm{~mm}$ and average dimensional deviation is $0.051 \mathrm{~mm}$. There is a negligible dimensional deviation in both the wooden pattern and its casting and that can be covered by considering major compensating factors. The shrinkage rate of aluminum casting is approximately $1.45 \%$.

Microstructure of a wooden pattern was built more densely and extremely brittle as compared to ABS pattern. There was no porous structure and no sealing or coating was needed on the part surface. The average surface roughness of wooden pattern and the aluminum casting were measured as 0.61 and $6.7 \mu \mathrm{m}$.

The dimensions of hollow ABS pattern and its casting were measured and recorded. Dimensional distribution charts are plotted based on the above data as shown in Fig. $3.2 \mathrm{a}$ and b. From Fig. 3.2, it is observed that $80 \%$ of dimensional deviations of the ABS pattern fall within $\pm 0.3 \mathrm{~mm}$

Dimensional deviation between CAD model and wooden pattern is approximately $0.025 \mathrm{~mm}$. For aluminum casting $80 \%$ of dimensional deviation falls within $\pm 0.3 \mathrm{~mm}$ and average dimensional deviation is $0.045 \mathrm{~mm}$. There is a negligible dimensional deviation of both the wooden pattern and aluminum casting and that can be covered by considering major compensating factors. The shrinkage rate of aluminum casting is approximately $1.38 \%$.

Microstructure of ABS pattern is dense and not extremely brittle as compared to wooden pattern. There is no porous structure. It has a good surface finish. The average surface roughness of ABS pattern and its casting is about 0.71 and $4.7 \mu \mathrm{m}$ respectively. The casting obtained from the ABS pattern has no major defects and was cast exactly to the required dimensions.

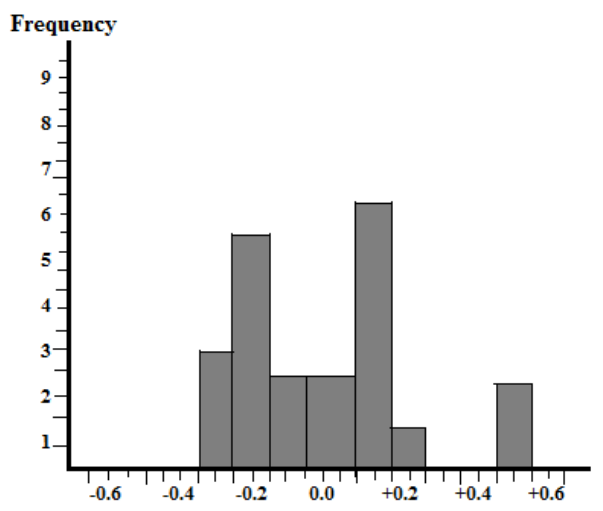

(a) Deviation from nominal dimension ( $\mathrm{mm}$ )

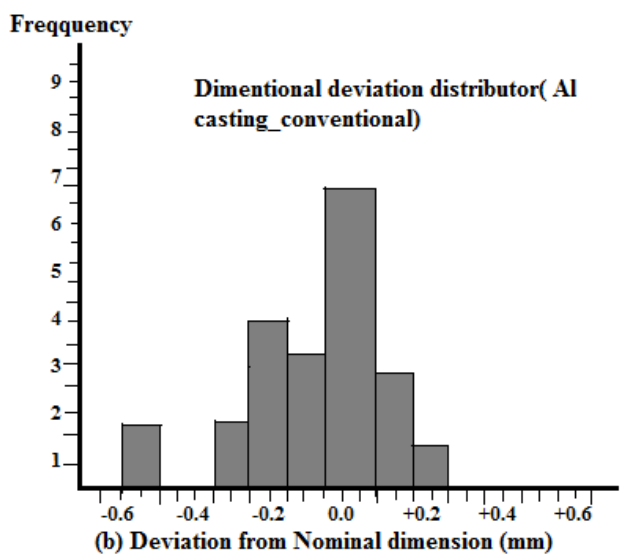

Fig -3.1 Dimensional deviation distribution chart for a) wooden pattern b) aluminium casting

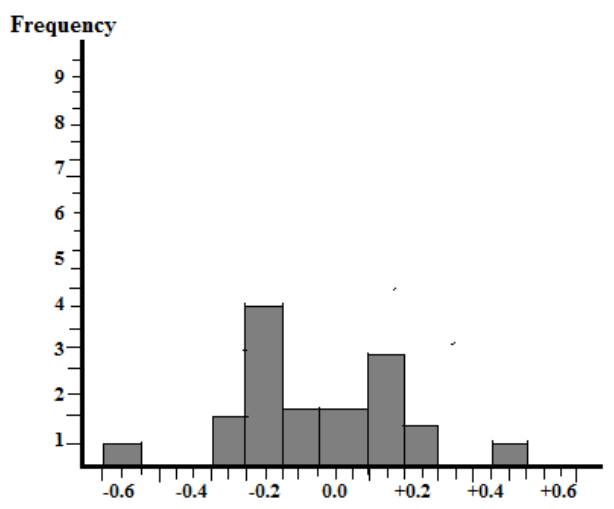

(a) Deviaion from Nominal dimansion ( $\mathrm{mm}$ )

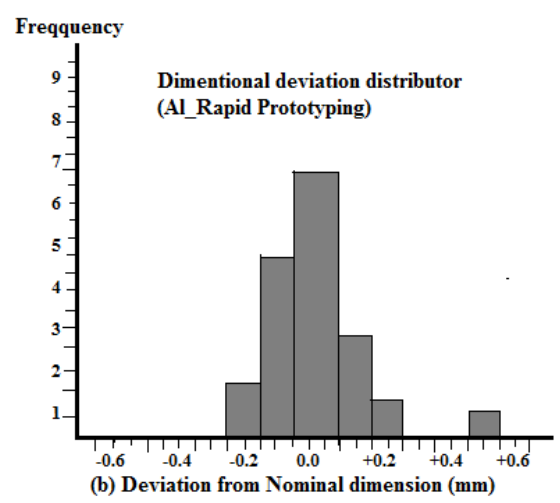

Fig -3.2 Dimensional deviation distribution chart of a) ABS pattern b) aluminium casting

\subsection{Cost and Manufacturing Lead Time}

Both manufacturing lead-time and cost investment studies were carried out on conventional and RP methods. The data obtained is shown in table 3.1 and 3.2.

The key limitation of employing wooden master pattern over FDM-fabricated patterns includes the long build time for the process to manufacture a part. For the manufacture of impeller model, the wooden master pattern took 22 hours while the FDM process took only 6.25 hours. The cost involved for manufacturing the wooden master pattern is about Rs 300 and for FDM pattern is about Rs 600 . 
Maximum replicate can be cast by using FDM pattern is around $130-150$ and by using wooden pattern 40-60 components can cast.

Table -3.1 Comparison of MLT of castings

\begin{tabular}{|l|l|l|}
\hline \multirow{2}{*}{ Particulars } & \multicolumn{2}{|l|}{ Manufacturing Lead Time (hr) } \\
\cline { 2 - 3 } & RP Method & Conventional Method \\
\hline CAD model & 4 & 4 \\
\hline $\begin{array}{l}\text { Hollow ABS } \\
\text { pattern }\end{array}$ & 2.25 & 18 \\
\hline Total time & 6.25 & 22 \\
\hline $\begin{array}{l}\text { Man power } \\
\text { (Nos) }\end{array}$ & 1 & 2 \\
\hline
\end{tabular}

Table -3.2 Comparison of Cost of castings

\begin{tabular}{|l|l|l|}
\hline Particulars & Cost \\
\cline { 2 - 3 } & $\begin{array}{l}\text { RP } \\
\text { Method }\end{array}$ & Conventional Method \\
\hline FDM pattern & 600 & 300 \\
\hline Max. replicate & $130-150$ & $40-60$ \\
\hline Total cost & 600 & 300 \\
\hline
\end{tabular}

\section{CONCLUSIONS}

Following conclusions can be made from this research work

1. The casting obtained from the RP method has more dimensional accuracy and high surface quality

2. The pattern made by ABS material can be used to produce more number of castings.

3. RP method involves less Manufacturing Lead time and nominal investment to produce patterns.

4. Patterns with complex shapes can be built easily

\section{REFERENCES}

[1]. Kalpakjian S Manufacturing processes for engineering materials, 2nd edn. Addison-Wesley, New York, 1991.

[2]. Lee CW, Chua CK, Cheah CM, Tan LH, Feng C Rapid investment casting: direct \& indirect approaches via fused deposition modeling. Int $\mathbf{J}$ Adv Manuf Technol 23(1-2), 2004, pp 93-101

[3]. Chua CK, Leong KF, Lim CS Rapid prototyping: principles \& applications. World Scientific, Singapore, 2003 [4]. Greenbaum PY, Khan S, Direct investment casting of rapid prototype parts: practical commercial experience. Proceedings of 2nd European Conference on Rapid Prototyping, Nottingham, UK, 15-16 July 1993, pp 77-93, [5]. Greenbaum PY, Pearson R, Khan S, Direct investment casting of RP parts: practical commercial experience. Proceedings of 4th International Conference on Rapid Prototyping, Dayton, OH, 14-17 June 1993 pp 43-50,

[6]. Dickens M, Stangroom R, Greul M, Conversion of RP models to investment castings. Rapid Prototyping J 1(4), 1995, pp 4-11.

\section{BIOGRAPHIES}

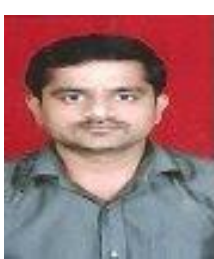

Mr. Sagar M Baligidad is working as an Assistant Professor in Mechanical Engineering Dept. of CMR Institute of Technology. Bangalore, Karnataka, India. He was awarded his Master of Technology Degree from "Jnana Sangama" VTU Belgaum. He is pursuing his $\mathrm{PhD}$ from VTU, Belgaum. He has 2 years of research and 3 years of teaching experience. He has more than 04 international/National conferences. His area of interest Rapid prototyping, collaborative manufacturing \& Alternative energy.

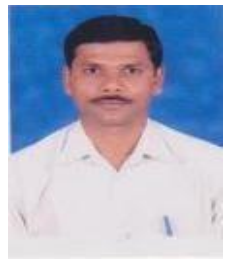

Dr. N Krishnamurthy obtained Ph.D. from VTU Belgaum. He is currently working as Professor in Mechanical Engineering Dept. of CMR Institute of Technology Bangalore, India. He has 1 year industrial, 8 years of research and 17 years of teaching experience. He has more than 8 research publications in international journals and more than 13 International/National conferences. His area of interest composites, thermal barrier coatings, alternative energy and advanced manufacturing technique.

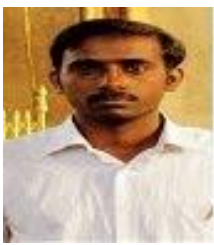

Mr. N Narendra is working as an Assistant Professor in Mechanical Engineering Dept. of CMR Institute of Technology. Bangalore, Karnataka, India. He was awarded his Master of Technology Degree from MVJ college of Engineering, Bangalore, India. He has 2 years of industrial and 3 years of teaching experience. His area of interest Rapid prototyping, collaborative manufacturing \& alternative energy.

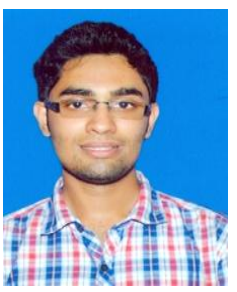

Mr. Ajay Srinivasan $\mathrm{N}$ is pursuing his bachelor of Engineering from CMR Institute of Technology Bangalore, India. His areas of Interest include Rapid prototyping, IC Engine and alternative energy. 